\title{
FORMATION DES FISSURES PRIMAIRES DE L'ECORCE TERRESTRE
}

\author{
Matthias Matrschinski
}

$\$ 1$. Fissures primaires et fissures secondaires. Fissures de premier type at de seconde type. Considerations générales. -.- Dans tout ce qui suit, nous distinguons entre fissures primaires et fissures secondaires en introduisanl ces expressions dans le sens suivant. Nous appliquons l'expression fissure primaire aux fissures apparues et en grande partic formécs sans influer (ou presque sans influer) les unes sur les autres. Elles surgissent sous l'influence de forces encore presque uniformes sur coute l'écorce terrestre. Le fait qu'elles commencent à se former en tel ou tel endroit est déterminé par la distribution non-uniforme rle la solidité de l'écorce liée aux variations locales rle son ipaisseur ou de ses propriété physiques. Au contraire la naissance des fissures qui surwissent apros la lormalion de ces fissures primaires est due aux iniøalité de la disposition téométrique des forces. En fait, lorsque quel'ques fissures sonl formées, les forces ne peuvent rester uniformément distribués sur l'écorce, - les parties de cette dernière au voisinage des fissures son dijà déchargées en direction perpéndiculaire aux fissures, tandis que dans d'autres partics de l'écorce les forces peuvent onrore augmenter. C'est pourquoi il convient de nommer toutes ces fissures dont la formation est due à l'existence des fissures primaires, fissures secondaires. Elles peuvenl apparaitre soil sous forme de bifurcation simultanées (fig. la), ou de hifurcation retardées (fig̨. 1)) soit même sans toucher, au premier moment, d'autres fissures (fig. lc). Au contraire le cas de la fig. ld représente les fissures primaires.

Du proinı de vue des applications (en géograplie, en géoplyysique, en réoloøie, elc.) ce sont les fissures primaires qui determinent les Frands traits de l'écorce tcrrestre, comme dans toute autre écorce (bomhes volcaniques "à crô̂te rle pain ", corps cxpérimentaux, etc.). Dur point de vue matlématique ce sont aussi les fissures primaires dont la théorie ent de beaucoup la plus simple en raison de la symétric approxinative des forces qui provoquent ces fissures, tandis que les fissures secondaires sont provoquies par des forces essentiellement asymétriques. C'est pourquoi pour decrire l'état au moment de la nais- 
sance des fissures primaires, on peat introduire, comme premiere approximation, la condition de symétrie splérique lans le: équations du

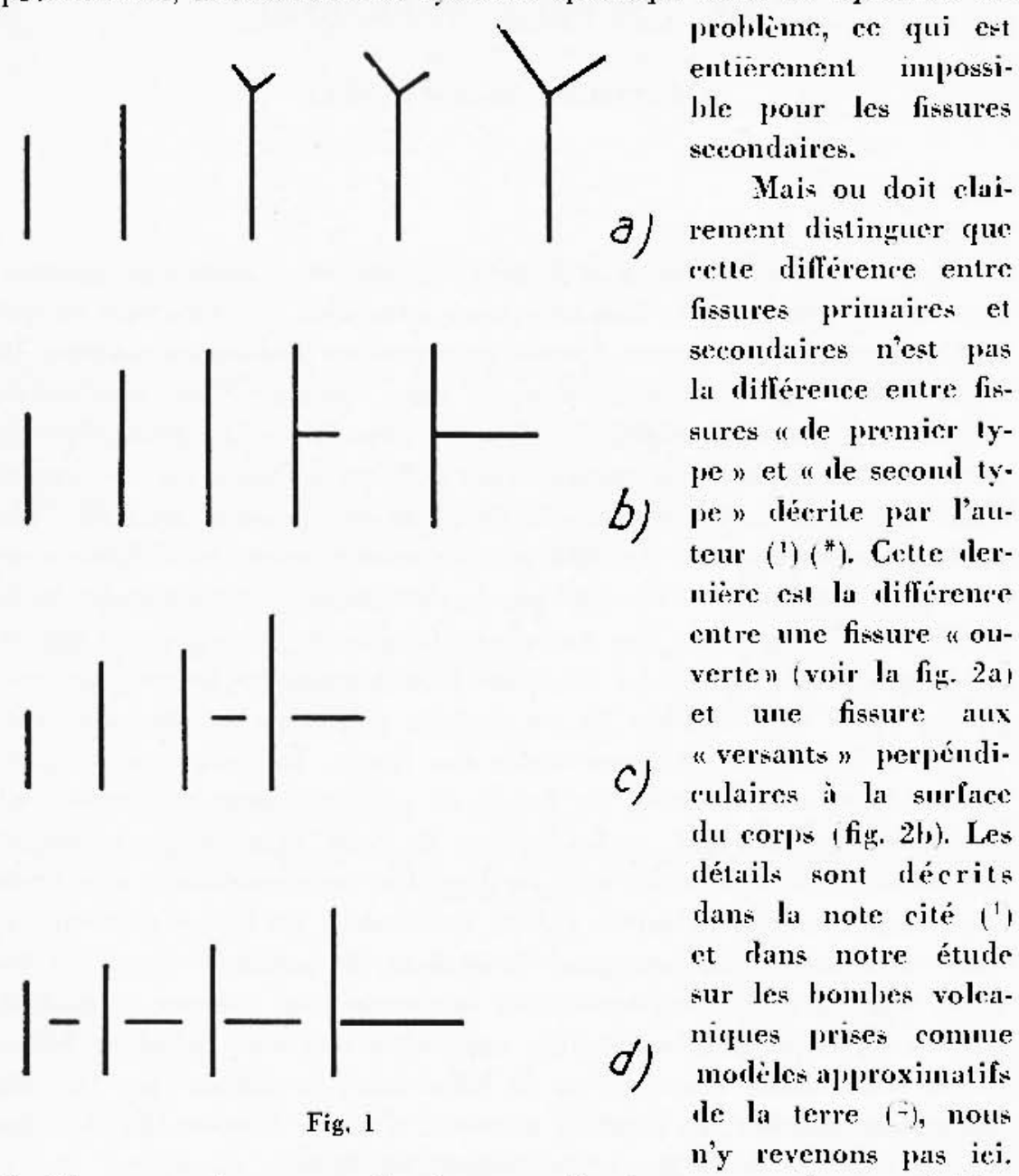

Soulignons seulement que les fissures primaires peuvent être aussi hien du premier que du second type, comme on le verra plus loin. La même chose est vruic des fissures secondaires.

(*) Dans la note citée $\{1$, décrivam les bombes volaniqutes aver les fissures de ces deux types, nous avons appliyue l'expression "la fissure printaire" pour "la fissure de premier type " et l'expression "la fissure secondaire " pour celle "de second type". Ici nous préférons employer seulement les expressions "de premier type" el "de second type" pour indiquer la différence des propriètés des fissures au sens de lia note (l), el conserver les expressions "printaire ", "secondaire" pour la distinction liée à leepoque de l'apparition des fistures, conume nous venons de les definir au début du paragraphe. 
Pour beaucoup de raisons prarni lesquelles la plus important est le nécessité d'appliquer des mélhorles mathematiques très différentes, il convient de considterer séparement le cas des fissures primaires et cehii des fissures secondaires. E1 dans la présente étude nous ne consiclérons que les primaires.

I] est clair que prima causa de ce pliénomène de fissuration est le regime olurmigue du glohe terrestre: c'est la distribution des lempératures, qui déterminc les tensions eventuelles: fans ces tensions l'appari-
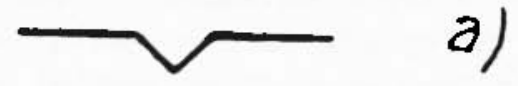
tion de fissures est inimarimable. Or, les mesu. res directes ne nous donnent que la distrilution des temjératures dans la eonche d'epaisseur minime en comparaison aves: les 6000$) \mathrm{km}$ environ du rayon terrestre. Beaucoup d'essazis t Jeeriefutes ont été

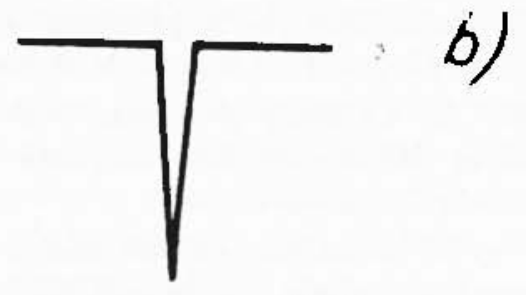

Fig. 2

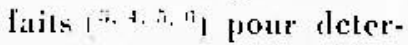
miner celle distribution à l'aide de telles ou telles autres domécs d'olservation indirectes. Les plus connues sont les six résultats sujvants: I. I, courhe de Holnes ${ }^{7}$ h, la plus ancienne peut-être, qui ait encore conservé de l'interét de nos jours: II. La courlie d'Adams ("): III. Citle de Jeflreys (") appliqucé par son atuteur au problème de déformations, ${ }^{11}$ et developpe après lui far Bullarel (12/; IV. De

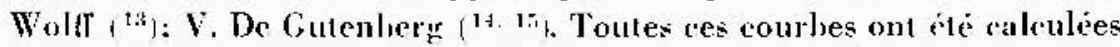
en partant des considerations purement mécanique et thérmiques: li où la radioactivití a éti prise en consicleration on l'a pris seulement en gualiti d'un gínirateur de chalcur. La demiere possibilite Vl possibilite d'un calcul hase sur les considérations de la théorie de la conductibilité électrique existe - elle est fonclée sur les risultats de Clopman ("H, the des collaborateur et recemment reprise par Rikitake $\left(^{17}, 14\right.$. Birch $\left({ }^{19}\right)$ et Verhooren $\left.2^{20,21}\right)$ ac sont oceupes de la distribution sulecrustale des températures. En liaison avec la dernière publiration de Verhooren on doit citer Valle (22.23). La mithode de

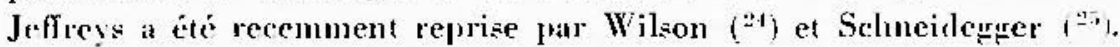
Le problime des tensions (probleme qui consiste a caleuler les tensions d'après tellc ou telle autre distrilution atuiscl a été également 
le sujet de nombreuses publications, qui commencent par celles de Davison C. $\left({ }^{26}\right)$ et Darwin G. $\left({ }^{27}\right)$ faites encore en XIX siècle. Parmi les contemporains citons Jeffreys $\left({ }^{11,28}\right)$ (modèle très connut), Conlomb $\left({ }^{211}\right)$ (sphère du liquide visquenx sans l'écorce), Sclineideguer $(-1)$ (écorce incompressible et noyau compressible). En liaison avec tout ce problème des températtres et des tensions on doit encore mention-

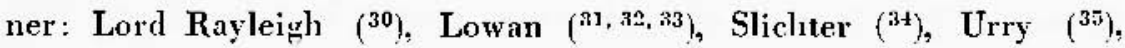
Gentry $\left({ }^{36}\right)$, Kuhn el Rittmann ( $\left.{ }^{37}\right)$, enfin Umbgrove $\left({ }^{38}\right)$. On peut en avoir un aperȩu dans le livre très intéressant de Wasiutinski $\left.{ }^{(39}\right)$. Mais inalheureusement tous ces résultals différent sensiblement les uns des autres. Ce n'est pas étonnant, car - faute de mestres directes ils sont basés sur des hypothèses fortuites et très différentes. De plus, on ne doit pas oublier que le problème se complique essentiellement, si on se place au point de vue de la théorie de courants sulocrustaux, theorie dont les éléments on trouve déjà clıez Pekeris $\left({ }^{40}\right)$ et qui at ité largement développe dans sa partie unathematique et wéophysiIte par Vening Meinesz if1. 12\}.

Pour toutes ces raisons on doit essayer de revoir toute la question en s'efforçant d'éliminer les lypollìses intermédiaires sur la distribution des températures dans l'intérieur du Globe.

Pour la description completement exacte du processus de refroidissement de l'écorce et de formation des fissures, ce sont les équations thermocinétiques sur lesquelles on doit se baser. Ces équations remuissent en un seul système les équations de la mécanique et celles de la théorie de lat conductililité de la chaleur: ce système est très compliqué et même non-linéaire. Cependant se ne sont pass seulement les difficultes de resolution du système des iqquations thermocinétiques qui nous forcent à introduire des simplifications dins ce système, mais surtout des considerations purement thioriques et générales. En effot un tel système contient entre autres de très nombreux états qui ne se réalisent jamais dans les cas qui nous intéressent.

De plus, il n'est pas toujours convenable de prendre en consideration même les états et les distrilutions qui se réalisent. C'est le théorème bien connu de Stokes $\left({ }^{43}, 4\right)$, qui nous permet de nous limiter dans nos considérations ì l'ícorce proprement dite et à la couche peu épaisse des parties liquides sousjacentes. Dans les cas stationnaires ou duasi-stationnaires - el comme on le verra, nous nous bornerons plus bas a de tels cas -... le théorìme de Stokes est applicable dans sa forme classique. De fait, l'équation de la conductibilité de cloaleur se dégénère dans ces cas el prend la forme d'une simple équation poten. 
tielle: $\Delta T=0$ (voir l'alinéa suivant). Grâce à celte forme simple ( $\Delta T=0$, on peut laisser de côte différentes généralisations du théorème de Stokes et se baser ici simplement sur le théorène classique. Comme il est connu / voir $\left({ }^{44}\right)$ /, la théorème de Stokes prouve que n'importe quelle redistribution des sources thermiques (les masses les sources du potentieli ne change pas le champ des températures externes (du potentiel) sous les conditions: 1) que le flux total de la chaleur à travers la surface engloluant les sources n'ait pas varie et 2) que cette surface mêne (- surface isothermique) n'ait pas non plus changée pendant lesdites redistributions. Le flux total de chaleur passant dans le milieu ambiant dégaqué par la Terre est bien connu (*); beautoup mieu que la distribution des sources énerg̣étiques dans le noyau. Se borner aux domaines à quelques dizaines de kilometres au dessous de la surface est égralement becaucoup plus facile que faire des bỵpotluéses incontrôlables sur les températures des coucbes profondes du noyau. Alors, le dbéorème de Stokes nous permet d'ac. cepter pour instant des hypotheses completement arbitraires; si les conditions que nous venons de mentionner sont réalisées, le résultat du calcul sera exact. Pour cette raison nous avons dejà appliqué ce theoreme dans notre note ( ${ }^{\text {tr }}$ ), et nous agissons dans le même sens ici.

En considirant seulement l'écorte même et les couclıes proches sousjacentes liquiles, nous pourrons faire une autre simplification importante, notamment la simplification qui nous donnera la possibilité de considérer l'équation de Ia conductibilité de chaleur sous sa forme la plus simple $1 \Delta T=0$, mentionnie déjà cirdessus.

Considérons la fig. 3, représentant la coupe de l'écorce $00^{\prime}$ et des coucles sous-jacentes $A B, A^{\prime} B^{\prime}$, etc. Dans les couclses liquides ou semiliquides surgissent les courants de convection apportant la chaleur vers l'écorce. Eıanı donnée la masse spécifique differente de ces couches $A B, A^{\prime} B^{\prime}$, etc, et l'agitation thermique intensive durant le temps proche à la naissance de l'écorce fet c'est la période qui nous intèresse ici), la formation des courants de convection de la forme abcd est très peu probable; re sont les courants de la forme efgh qui se produissent $1^{* *}$. Ces courants ofgh peuvent être considérés, comme on le fait

(*) II ne sigit jas ini de fixer la valeur du flux que nous introduisons dans notre calcul: la valcur d'aujord'hui ou la valeur augmentée d'après telle ou tclle autre hypothese. Il šagit plus de fait gute, dans tous ces cas, on peut admettre une valcur constante pendant le tempe de la formation de fissures.

$1^{k \neq 1}$ Il est necessaire de souligner quavec cette remarque nous ne nous oprosons 
babituellement, comme quelique sorte des "tourbillons" de Benart , $^{\text {nii }}$.

Sans entrer dans le détail sur les formes el sur le caractire du mottvement de ces a tourbillons" ofgh, on peut remplacer le courant wlobal dû ì la convection dans l'espace CDEF à un vecteur dont la direction coincide avec celle de $r$. Dans le cas d'un corps sphierique łp. e. la Terrel cetle grandeur $r$ n'es] que le rayon. C'est pourcuoi dans un

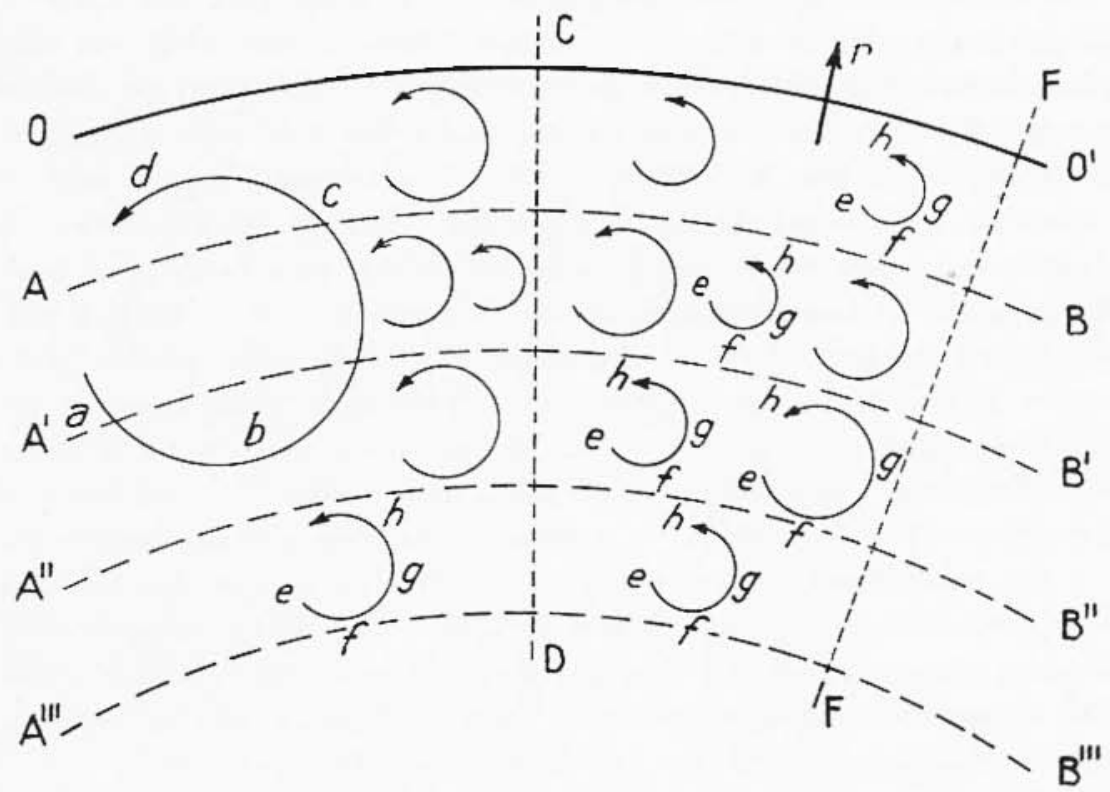

Fin. 3

corps sphérifue en voie de refroidissement les couranls de chaleur: 1) courant de convection globale et 2 s courant de conductibilite, coüncident en direction: le premier comme le second surivent les rayons de la sphère orientés vers l'extérieur. Soit $Q_{1}$ le premier, $Q_{\text {: le se- }}$ cond courant, $Q=Q_{1}+Q$, leur somme qui desermine la changemen de la tempirature:

$$
\operatorname{div} Q=c \frac{\partial T}{\partial i}
$$

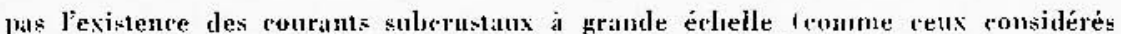
p.e. par Vesing Meisesz). Nous iusistons seulentent que l'effit du lrasport est du aux courauts de la fig. 3 , mais non aux rourants ayant des dimensions considérables.
} 
I $T$ temprirature, $t$ tem]'s, c chaleur spécificquei. $Q_{1}$ eet déterminé par le grradient thermométrique:

$$
Q_{1}=k_{1} \text { irad } T,
$$

O.: par la vitesse moyenne $v$ de la convedion:

$$
Q_{2}=c_{1} v ; \quad v=a \text { wrad }\left(\frac{1}{\varrho}\right)
$$

aii fo es la densité. Celte derniere détermince de nouveau par la tentprerature:

$$
\frac{1}{e}=b_{1} T+b
$$

nous prermet, dans le cas de la coüncidence des directions de $Q_{1}$ et $Q_{2}$, decrire l'expression pour le courant total:

$$
Q=Q_{1}+Q_{2}=\left(k_{1}+c_{1} a b_{1}\right) \text { mrad } T
$$

De cette expression [5] avec [1] on tire définitivement:

$$
c \frac{\partial T}{\partial t}=\operatorname{div}\left(k_{1}-f-c_{1} a b_{1}\right) \operatorname{arad} T / \rightleftharpoons\left(k_{1}+c_{1} a b_{1}\right) \Delta T
$$

irfuation de la conduclibilité, dans sa forme elassjque, aver la seule différence qu'au lieu de la combuctibilite $k_{1}$ on voit $l_{a}$ somme $k_{1}+c_{1} a b_{1}$ rxprimant la "conductibilité totale " par la conduchililité proprement dite el par la "conduetibilité " conventionnclle de la convection. Pour rette conductililité tolale nous introduisons la lettre $k$ sans indice. [l est facile de voir que prour la partie solicle fe'cst-a-dire pour l'écorce elle-mêmer la grrandeur $k$ se reduit à $h_{1}$, la convection n'existant pas. Aussi, hien que la ronchutilitité classique $k_{1}$ soil moins élevée pour les liquides que pour les solides:

$$
k_{\mathrm{1}, 1}<k_{1, \mathrm{~s}}
$$

il ch va lout autroment avec la grandeur $k$ :

$$
k_{1}>>k_{s}
$$

l'inégalités étant d'aukant plus marquice que l'agitation du liquide est plus intenser. Entin, en tward aux souress de chaleur dégievte dans le noyau, l'ípuation [6] s'écrira:

$$
c-\frac{\partial T}{\partial t}=k \Delta T+S(t, x ; y, z),
$$


où $S$ est l'intensité de ces sources. Pour les processus stationnaires, et quasi-stationnaires, d'aurès le théoreme classique de Stokes, il n'est pas nécessaire de chercher à étal,lir la vraie distribution des sources el des temperatures dans le noyau. Au contraire on peut prendre telle distribution qui facilite la résolution (voir $\left({ }^{45}\right)$ ). Nous ne revenons pas sur ce problème partiel ici en dépit de tout son intérét pro-

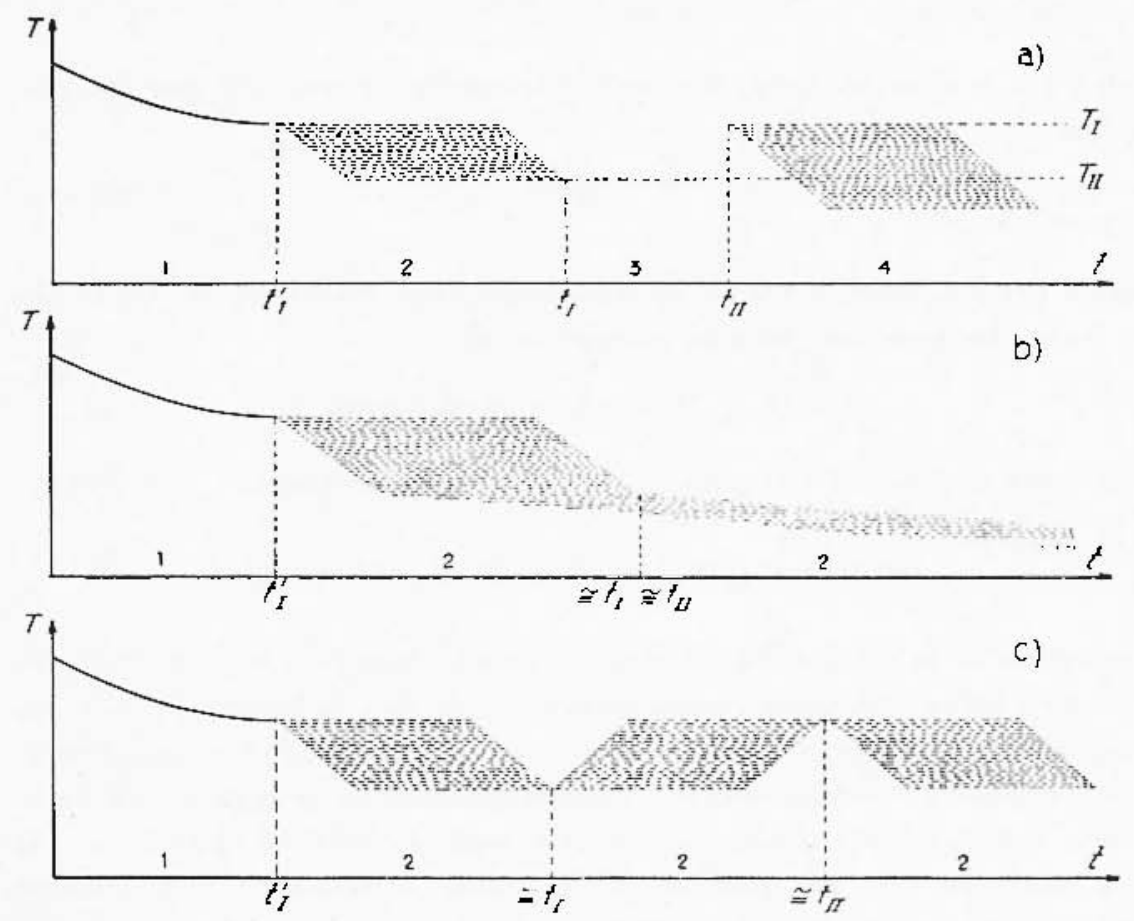

Fig. 4 - 1) Elats non stationnatices: 2) Etats quasi-stationnates; 31 Etat non stationnaires: +1) Etats quasi-stationnatires.

pre, parce que le vrai probleme de la formation des fissures est le problème des équations de la mécanique, où l'on doit introduire les changements de volume, déterminés par les changements de la température / selon [4] /. Fn ce qui concerne le calcul de la temperrature, ce calcul est lié à l'équation [9]. Mais is cause de l'extrême lenteur de ces changements le processus thermique n'est que le processus quasi-stationnaire ou presque stationaire. Par l'expression "quasi-stationnuire" nous entendons le processus compose des processus purement stationnaires, c'est-à-dire, le processus de changrements tellement lents que à chaque instant donne la distribution des 
températures est une distribution stationnaire, en dépit de ces clrangentents. Ce processus quasi-stationnaire peut être illustré, pour le cas du refroidissentent de la Terre, par les figs. $4 a$, $4 \mathrm{l}$ et $4 \mathrm{c}$ où trois variantes possibles de ce pluénomène sont représentées. La fig. 4a est empruntée à notre note (15).

Pour un tel processus quasi-stationnaire la distribution particu. lière de la température sera donnée par l'équation [9] où $\frac{\partial T}{\partial t}=0$ de façon très exacte. Mais le changement avec le tempz reste indéterminable à l'aide de [9], it n'est détcrminé que par le changennent de $S$ avec Ic temps. Au cas où $S$ est provoqué par l'activité radioactive, ce changement est de la forme d'unc fonction exponcntielle, en cas d'autres bypothesies, il sera de telle on telle autre forme plus conntiquée. Nous n'entrons pas ici dans tout ce détail; ces questions sont étudiées aillcurs. Ici nous prenons ce cluangement de la température comme una fonction donnèe: fonction à variation extrêmement lente.

On peut aller encore plus loin dans la voie des sintplifications. Comme un ras linite parani les possibilités de traiter notrc problème d'une facon thermocinétique (voir $\$ 3$ i on peut considérer le cas d'une sphère, dont le processus de solidification consiste en une suite d'états non seulement thermocinétiquement stables mais, plus simplement encore, isotherniques. Dans un tel cas il s'agit d'une solidification infinement lente, tous les courants de chaleur étant infiniment petits, et la seule grandeur thermique qui ne doit jas ètre négliquée est le chanrement de volunte au point de fusion.

Certes, la solidification se propage en réljtí aver une vitesse finie domnant lieu à des courants de chaleur qui ne sont pas infiniment pelits; mais, à titre de decription approximative des processus lents, le cas de la solidjfication à temperaturc constante peut être intéressant.

Ce cas ne se réalise naturellement pas pour la Terre, mais it est facilentent ríalisable par l'expéricnce et de plus il est une réalité pour ccrtaines hombes volcaniques. Enfin. ce jrocessus "jsothermique" présente les moindres difficultés du calcul. Nous le considérons en détajl faus le paragraplue sujvant $(\$ 2)$. Dans le $\$ 3$ nous revenons au cas plus grénéral d'un processus " quasi-stalionnairen.

\$ 2. Refroidissement "isothermique". Formation des fissures du premier type. - Nous avons déjà parle plus haut des "équation de la mécanique " qui doivent être appliquécs. La méranique des corps solicles connaît deux esjéces de colles cquations: équations de l'élasti- 
cité et équalions de lat plasticité. Tandis que les premières sont applicalbles sans conditions speciales l'application des secondes est inseparalslement liée à la condition dite de Tresca $\left({ }^{47}\right)$-Saint-Venan $\left({ }^{48}{ }^{49}\right)$ dans sa forme ancienne ou dans la forme donnée par IIuber (17) . Mises $1^{+19} \cdot{ }^{40}$. Quelle qu'en soit la forme, cetle condition exige toujours soit un état très tendu soit un étal sous grand pression. Aux époques voisines de la naissance de l'écorce terrestre tel n'est pias le cas. C'est seulement longtemps aprìs qu'on peut attendre les pressions

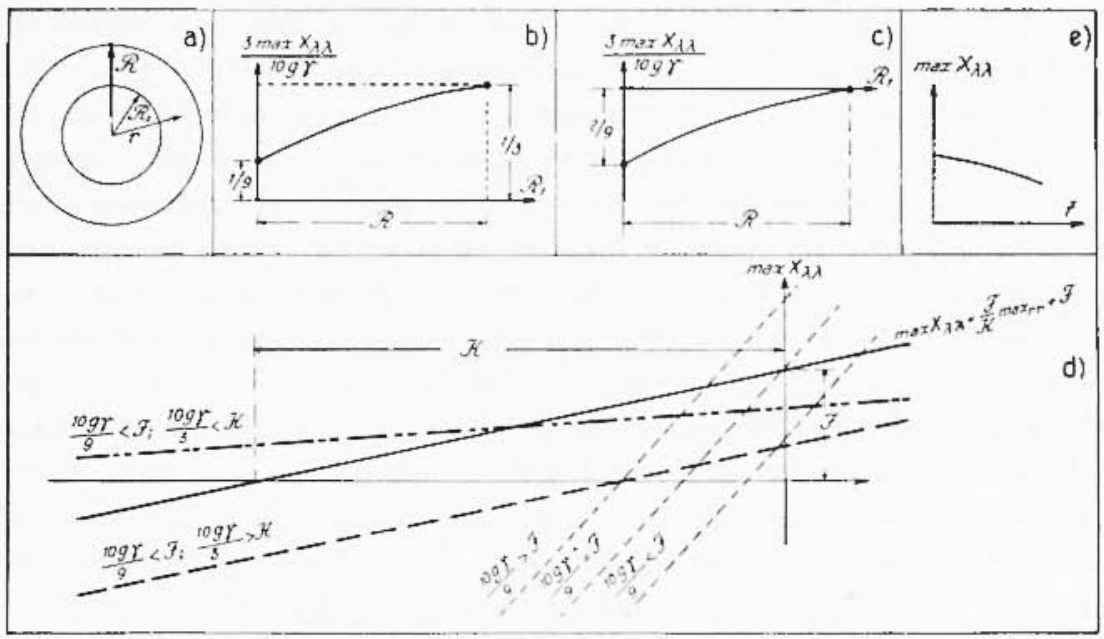

Fig. 5

ou les tensions. De plus, les roches ne peuvent ètre tendues plistiquement, elles se rompent avant de couler. Ce n'est que sous la pression de tous côtés que les roches coulent. Pour toules ces raisons il convient pour traiter la question de la fissuration, de se limiter à l'emploi des éfuations de l'élasticité; aux premiers stades elles sont seules applicalbles. Certes, lors de l'apparition des yrandes pressions les équafions de la plasticité doivent être ajouties; mais, comme nous le verrons, ce sont des tensions, et non pas des pressions qui alpraraissent.

Partons donc des équations de l'élasilicité, mais supposons de surcroît que la partie solidifice idécrite par ces equationsl a diminué à la solidification de la valeur $\ddot{*}$, e'est-it-dire que le volume $F$ (avant la solidification d devient après elle le volume $V(1-\%$.

Considérons le moment où la solidification de la sphère de rayon $R$ a atleint les points se trouvant à la distance $R_{1}$ du centre, lo reste 
de la sphire élan encore liculide (voir la fig. 5a). Si la cavilé sphérique du rayon $R_{1}$, remplie en fait par un licquide, étail vide, l'écorce diminuerait dans le rapporl $\because$. Mais en fait cette écorce, étant remplie par un liquicle peu compressible, sera tendue par les prossions très élevée qui peuvent so produire dans cetle dernière. Il est évident que pour celle sphire liquide de rayon $R$, on a:

$$
u_{1}=\frac{\rho_{1}}{3 k} \frac{R_{1}}{3 k}
$$

où $u_{1}$ est le déplacement de la surface de cette sphère provoqué prar la pression $p, k$ le module de la contpressibilité de volume.

Nous n'écrivons pas lás écpuations de l'é]asticití sous leur forme complète fvoir, p. e. ('Tll. D'apres la remarque du 1 s sur l'étude des fissures primaires, on peut introduire les conditions de la symétrie spliérique, qui, appliquée aux érfualions de l'é]asticité, pernettent de transformer ces demières dans le systène $\left(^{*}\right)$ suivant:

$$
\begin{gathered}
\frac{\partial}{\partial r}\left(r^{2} X_{\mathrm{rr}}\right)=2 r X_{\lambda .,} \\
X_{\mathrm{rr}}=2 G\left(\frac{\partial u}{\partial r}+1 / 2 \theta\right) ; X_{\lambda \lambda}=X_{\mathrm{v} 0}=2 G\left(\frac{u}{r}+\frac{1}{2}-\theta\right) \\
\theta=\frac{1}{r^{2}} \cdot \frac{\partial}{\partial r}\left(r^{2} u\right) ;
\end{gathered}
$$

d'où il s'ensuit successivement:

$$
\begin{gathered}
r^{\circ} u^{\prime \prime}+2 r u^{\prime}=2 u \\
u=C_{1} r+\frac{C_{2}}{r^{2}}
\end{gathered}
$$

Puisque l'on avait exprimé la position de la limite clu liquirle non comprimé en coordonnées déplacées de l'écorce, le déplacement de celte posilion sera ividemment $R_{1} ; 3$; le déplacement provoqué par

(*) Jjans ce systeme le couflicient de Poisson $m$ est pris égal à 1 ce que esl trés prorlie i li1 valeur moyerue pour les roclies - exuations (3). - IJe plus, les forres de pesanteur ont éte ontises — equation $\{21$, parce que l'influence de poids est minime pour les phénouénes de refroidissement et de lissuration de corps expérimentaux, meine ayant des rayons jusquaux rentaines de metres. On ne peut niturellement négliger rettre intlueme pour les rorps relestes el pour la Tcre. En considerant pette dernière, nons introduirons dans $\$$ t la correction provenant de l'action de poids. 
la compression sera [1]; ainsi la position de la limite du liquide comprimé devra coïneider avec le déplacement de l'écorce à cette limite:

$$
R_{1} / 3+\left.u_{1}\right|_{r=R_{1}}=u \mid r=R_{\mathrm{l}}
$$

Les pressions à cette limite étant aussi égales:

$$
\left.p_{1}\right|_{r=R_{1}}=X_{r} \mid r=R_{1}
$$

les formules [1] et [7] nous amènent ì la première condition de la limite:

$$
R_{1} \gamma / 3+\frac{R_{1}}{3 k} X_{z 1} \mid \begin{aligned}
& r=R_{1} \\
& r=R_{l}
\end{aligned}
$$

La deuxiène condition est évidente:

$$
\left.X_{\mathrm{rr}}\right|_{r=R_{1}}=0 .
$$

En introduisant l'abrévation:

$$
\frac{G}{3 k}=\varepsilon
$$

on obstient de [10]

$$
5 C_{1}-\frac{4 C_{2}}{R^{3}}=0 ; \quad C_{2}-R^{3} \frac{5}{4} C_{1} .
$$

et de [9]:

$$
\begin{aligned}
& C_{\mathrm{t}}=\frac{4 R_{1}{ }^{3} \gamma}{3 / 4 R_{1}{ }^{3}+5 R^{3}+20 \mathrm{E}\left(R^{3}-R_{1}{ }^{3}\right) /} \\
& C_{0}=\frac{5 R^{3} R_{1}{ }^{3} \gamma}{3 /+R_{1}{ }^{3}+5 R^{3}+20 \varepsilon\left(R^{3}-R^{3}{ }^{3}\right) /}
\end{aligned}
$$

Maintenant à l'aide de [6] on a les formules pour le déplacement conditionnel :

$$
u=C_{1} r+\frac{r_{z}}{r^{2}}=\frac{z_{-1}-\frac{4}{3}}{4 R_{1}{ }^{3}+5 R_{1}{ }^{3}+20 \varepsilon\left(\frac{r}{R_{1}}\right)+5 R^{3}\left(\frac{R_{1}}{r}\right)^{2}{ }^{2}} ;
$$


et joul le déplaccoment ríel:

$$
u_{\text {Intul }}=-\frac{R_{1} \gamma}{3}+u .
$$

Ensuite de [3] on tire:

$$
X_{r r}=\frac{G \gamma}{3} \frac{20 R_{t}{ }^{3}}{4 R_{1}{ }^{3}+5 R^{3}+20 \varepsilon\left(R^{3}-R_{\mathrm{l}}{ }^{3}\right)}\left[1-\left(\frac{R}{r}\right)^{3}\right] ;
$$

avec les corollaires:

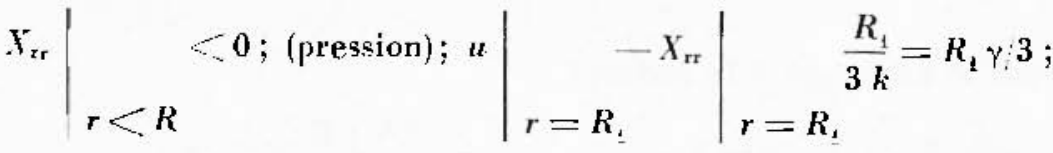

$$
\begin{aligned}
& Y_{r \mid}=0 ;
\end{aligned}
$$

(le premier montre que $\boldsymbol{X}_{\mathrm{rr}}$ est la pression) et:

$$
\begin{gathered}
X_{\lambda \lambda}=X_{i v}=G\left(5 C_{1}+\frac{r^{\prime}{ }_{2}}{r^{3}}\right)= \\
=\frac{G_{\gamma}}{3} \frac{10 R_{1}{ }^{3}}{4 R_{1}^{3}+5 R^{3}+20 \varepsilon\left(R^{3}-R_{1}^{3}\right) !}\left[2+\left(\frac{R}{r}\right)^{3}\right)>0 ;
\end{gathered}
$$

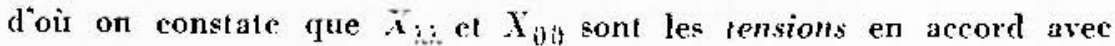
lévidence.

Dans toutes ces expressions la qrandeur [11] est très petite, étant donné que la compressiluilité des lituides est négligeable en comparaison de la compressibilité des corps solides. Ainsi pour simplifier lanalyse, peuton commencer l'élucle des formules [17] et [19] en partant du cas d'un liquide incompressible $(k=\infty, \varepsilon=0)$. Ici la formule [17] se.transforme en:

$$
\left.X_{x}=\frac{20 G_{Y} R^{a}}{3\left(5 R^{3}+4 R_{1}^{3}\right)}\left[1-\frac{(R}{r}\right)^{3}\right]
$$

(le maximum de $X_{\mathrm{rr}}$ at moment donné sera

$$
\left.\max X_{r_{\mathrm{r}}}\right|_{r=R,}=\frac{20 G \gamma\left(R_{1}^{3} \cdots-R^{3}\right)}{3\left(5 R^{3}+4 R_{1}^{3}\right)}<0 ;
$$


au point $r=R_{t}$, et la formule [19] se simplifie en:

$$
x_{\lambda \lambda}=x_{00}=\frac{10 G \cdot R^{3}}{3\left(j R^{3}+4 R_{1}^{3}\right)}\left[2+\left(\frac{R}{r}\right)^{3}\right] .
$$

Le maximum de cette tension sera toujours au fond de l'écorce tau point $r=R_{1} ;$ il sera éral à:

$$
\left.\max X_{7.2}\right|_{r=R_{i}}=\left.\max X_{03}\right|_{r=R_{i}}=\frac{10 G \gamma\left(2 R_{1}^{3}+R^{3}\right)}{3\left(5 R^{3}+4 K^{3}\right)}>0 ;
$$

ayant le minimum à la surface:

$$
\left.\min X_{\lambda \lambda}\right|_{r=R}=\left.\min X_{00}\right|_{,=R}=\frac{10 G \gamma R_{1}{ }^{3}}{5 R^{3}+4 k_{1}{ }^{3}}>0 .
$$

Ces résultats / $[20],[21|| 21 a \mid$, et $|22|$ / sont représentés sur les figlres $5 \mathrm{l}$ el $5 \mathrm{c}$.

Enlin, pour le déplacement total on a dans ce cas:

$$
u_{\text {1olul }}=-\frac{5 \gamma R^{3} R_{1}}{3\left(5 R^{3}+4 R_{1}^{3}\right)}\left[\frac{r}{R_{1}}-\left(\frac{R_{i}}{r}\right)^{2}\right]
$$

expression qui a les propriétés évidentes:

$$
\begin{aligned}
& u_{\text {tolat }}=0, \text { si : } r=R_{1} ; \\
& u_{\text {total }}<0, \text { si : } r>R_{1} .
\end{aligned}
$$

En revenant au cas le plus généval on doit étudier les grandeurs:

$$
\begin{aligned}
& \left.\max X_{\lambda \lambda}\right|_{r=R_{1}}=103 G_{Y} M\left(R_{1}\right) ; \\
& W_{1}(R)=\frac{2 R_{1}{ }^{3}+R^{3}}{4 R_{1}{ }^{3}+5 K^{3}+20 \varepsilon\left(R^{3}-R_{1}^{3}\right)} ; \\
& \left.\min X_{\gamma . j}\right|_{r=R}=10 / \& G_{Y} m\left(R_{1}\right) \\
& m\left(R_{1}\right)=\frac{3 R_{1}{ }^{3}}{4 R_{1}{ }^{3}+5 R^{3}+20 \varepsilon\left(k^{3}-K_{1}{ }^{3}\right)} .
\end{aligned}
$$


Elant ronné qu'au voisinage de $R_{1}=R$ on peut écrire:

$$
\delta=R-R_{1}, \quad R_{1}^{3}=R^{3}-2 R^{n} \delta,
$$

il est très facile de se persuader que:

$$
\left.h_{1}(R)=\frac{3 R^{3}-4 R^{-} \delta}{9 R^{3}-8 R^{*} \delta+20 \varepsilon \cdot 2 R^{2} \delta}=1 / 3[1+\text { les termes négatifs })\right]
$$

En general on a toujours:

$$
H_{1}(K)<\frac{2 R_{1}{ }^{3}+h^{3}}{4 R_{1}{ }^{3}+5 R^{3}}=\left.\frac{3}{10 G_{Y}} \max X_{j, \lambda}\right|_{r=R_{1}} ;
$$

sauf le cas où $R_{1}=R$; là on a: $M_{1}(R)=1 / 3$. Mais revenons au cas du liquide incompressible.

Avant rle tirer les conclusions des résultats $[20 \mathrm{a}]$ et $[21 \mathrm{a} \mid$, il est inléressint de faire encore une remarque.

Ayant calculé la derivée $\frac{d\left(\max X_{h 7}\right)}{a\left(\max \ddot{X}_{\mathrm{rr}}\right)}$ à l'aide de:

$$
\frac{d\left(\max X_{\lambda, \lambda}\right)}{d\left(\max X_{\mathrm{rr}}\right)}-\frac{d\left(\max X_{\lambda, \lambda}\right)}{d(R, / R)}: \frac{d\left(\max X_{\mathrm{rr}}\right)}{d(R, R)}=13,
$$

on oblient une relation très simple:

$$
\max \ddot{X}_{1,2}=1,3 \max X_{j, \lambda_{2}}+\frac{10 G_{Y}}{9}
$$

(voir la fig. $5 \mathrm{dl}$. Introduisons les valeurs critiques de $\boldsymbol{X}_{\lambda . \gamma}$ et $\boldsymbol{X}_{\mathrm{rr}}$ autquelles commencent les ruptures et les écoulements:

$$
\text { c:it. } X_{j i} \text { et crit. } X_{\mathrm{rr}} \text {. }
$$

Les grandeurs [31] ne sont ni constantes, ni indépendlantes: ce sont seulement les grandeurs:

$$
F=\text { crit. }\left.X_{M}\right|_{X_{\mathrm{rr}}=0} \text { et } K=\text { crit. } X_{\mathrm{rr} /} \mid X_{\lambda, .}=0
$$

qui jưluent être considérées comme les constantes de matière (en dipit ru'clles ne sont pas, elles non plus, les constantes physiques dans 
le sens propre). Les conditions de Tresca - Saint-Venant ou Huber Mises ne s'appliquent pas aux élats tendus des roches (voir \$ 1), il convient d'introduire une relation approximative entre les deux wrandeurs $[34] ;$ p. e. la relation:

$$
\text { crit. } X_{\lambda \lambda}=\frac{F}{K} \text { crit, } X_{\mathrm{rr}}+F \text {. }
$$

Or, des données expérimentales:

$$
5 F<K<25 F
$$

on peut déduire que $[36 \mid$ et $|33|$ sont incompatibles qvoir la fig. Id). De toul cela on peut tirer les conclusions suivantes:

I. Lorsque:

$$
\text { max. } X_{\lambda i}=\frac{10 G \gamma}{9}>F
$$

les fissures du premier type se forment obligatoirement.

2. Lorsque:

$$
\frac{10 G Y}{9}<F, \text { mais } K<\frac{10 G Y}{3}
$$

la formation des fissures de second type est logicuement possible, mais en raison de l'incompatililité de $[36]$ et de $[33$ ] il n'existe pas de sulsstance constiluant l'écorce terrestre correspondant ì ce cas. Ainsi ce cas est physiquement impossible.

3. Lorsque:

$$
\frac{10 G_{Y}}{3}<F \text {, et } K>\frac{10 G_{\gamma}}{3}
$$

aucune fissure ne se forme.

Comme nous l'avons déjà dit, tout cela n'est directement applicable qu'aux corps pas très grands. Dans le $\$ 4$ une correction sera indiquéc qui est nécessuire dans les cas des corps ayant des rayon: de plus de 100 mètres. 
\$3. Refroidissememt "quasi-stationnaire". Formation des fissures du deuxis̀me type. - Revenons maintenant at cas plus général, ras d'un processus "quasi-stationnaire". Les températures considercées dans ce cas variant avec le temps, varient atssi d'un endroit à l'autre cl, ce qui est pour nous le plus important, elles ne sont pas les mêmes dans l'écorce el dans les parties liquides zousjacentes. P'our les calculer approximativement on peut appliquer les mêmes simplifications que celles de la note citée $\left.{ }^{15}\right)$ : l, la constance de la température extérieure: $T_{\text {ext. }}=$ const; 2 l la coïncidence de l'isotlierme $T=T_{\text {fus. }}$ foù

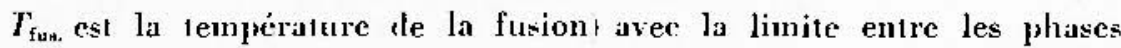
liquide et solide des roches; 3। l'applicabrilité de la loi simple de radiation sur la surface extérieure $\left(T_{0}-\right.$ la température sur cette sur(ace):

$$
T_{\mathrm{o}}-T_{\mathrm{cxi}}=\beta \frac{\partial T}{\partial r},
$$

4! la distribution presque linéaire des températures dans l'écorce naissante, permethant dappliquer les relations simples: pour la température moyenne de l'écorce

$$
T \geq \frac{T_{\text {fus. }}+T_{\mathrm{o}}}{2}
$$

pour le grarlient:

$$
\frac{\partial T}{\partial r} \simeq \frac{T_{a}-T_{\text {fus. }}}{\Delta R},
$$

et pour le changement de la température moyenne:

$$
\Delta T \cong \frac{T_{\text {ius. }}-T_{\mathrm{o}}}{2},
$$

déterminé par le changennent de $T_{4}$ (baisse en cas de refroidissement), etc. (voir la fig. 6ai).

A partir des expressions $|1|$ et $\lceil 3 \mid$ on obtient une formule déterminant la température sur la surface de limite

$$
T_{\mathrm{o}}=\frac{T_{\mathrm{ex} .} \cdot \Delta R-\beta \cdot T_{\mathrm{fur} .}}{\Delta R-\beta}
$$


et le changement de celle-ci:

$$
\unlhd T=\frac{\Delta R\left(T_{\mathrm{fud}}-T_{\mathrm{ex}}\right)}{2(\perp R-\beta)}<0 .
$$

Etant donné que l'épaisour de l'ecorce $\triangle R$ parte de 0 el reste toujours moindre que $\beta$, la valeur absolue de $\Delta T$ est approximativement égale à

$$
\Delta T /=\frac{T_{\text {sua. }}-T_{\text {ar. }}}{2 \beta} \Delta R=\frac{\gamma^{\prime}}{\alpha} \perp R
$$

oii :

$$
\gamma=\alpha \frac{\underline{T}_{\text {ius. }}-T_{\text {ex }}}{2 \beta} .
$$

C'est cetle valcur $\perp T$ qui provoque le changement themometrique du volume, s'ajoutant au changement de volume au point de fusion $(\gamma)$, voir le commencement du $\$ 2$. Cela revient à dire quau lieu de $\gamma$ de la théorie du $\$ 2$, il convient d'utiliser la grandeur totale:

$$
\gamma_{\text {lutul }}=\gamma+y^{\prime} \perp R \text {. }
$$

Etant clonné que le calcul avee $\gamma_{\text {tonle }}=\gamma$ es déjà réalisé (voir $\$ 2$ ), considérons pour simplifier les transformations mathematipues le cas contraire ytutals $=\gamma^{\prime} \perp R$. L'étal de l'écorec en voie de refroidisse. ment, - état d'un corps polycristalin et en partie même visqueux --, est tel que l'influence du terme $\gamma^{\prime} \Delta R$ prévaut sur l'influence du terme $\gamma$ - C'est pourquoi en supprimant ce dernier nous ne fitisons pas une faute essentielle. En outre il n'est pas difficile de traiter mathématiquement le problème dans la forme complete [8], mais ceci ne donne aucun résultat fondamentalement different, parce que la considération des deux cas extrèmes (cas du $: 2$ et celui-ci de $\gamma_{\text {iutale }}=\gamma^{\prime} \Delta R$ ) jetle une lumière suffisante sur les cas intermédiaires.

En introduisant dans notre calcul la valeur $\gamma^{\prime} \unlhd R$ au lieu de $\gamma$, nous ne changeons rien dl'essentiel dans les considérations el les trans. formations mathématiques du $\$ 2$. Avec ce rhangement, au lieu des formules $[17]$ et $[18]$ ilu $\$ 2$, nous trouvons:

$$
\boldsymbol{X}_{\mathrm{rr}}=\frac{20 G \gamma^{\prime} \backslash R}{3} \cdot \frac{R_{t}{ }^{3}\left[1-\left(\frac{R}{r}\right)^{3}\right]}{4 R_{1}{ }^{3}+5 R^{3}+20 k\left(R^{3}-R_{t}{ }^{3}\right) \mid},
$$


et :

$$
X_{\lambda i .}=X_{00}=\frac{10 G \gamma^{\prime} \Delta R}{3} \cdot \frac{R_{1} \div\left[2+\left(\frac{R}{2}\right)^{3}\right]}{\text { simile }}
$$

Supprimant cnfiu, comme nous lavons fait dans $\$ 2$, les termes avec $\varepsilon,-$ écs-à-dixe se liornant aux cas où le noyau est peu compressille, - on trouve:

$$
X_{\mathrm{rr}}=\frac{20 G \gamma^{3} \Delta R \cdot R_{1}^{3}}{3\left(5 R^{3}+4 R_{1}^{3}\right)}\left[1-\left(\frac{R}{r}\right)^{3}\right],
$$

et :

$$
X_{r^{3}}=X_{05}=\frac{10 G r^{\prime} \perp R \cdot R_{1}^{3}}{3\left(5 R^{3}+4 R_{1}^{3}\right)}\left[2+\left(\frac{R}{r}\right)^{3}\right] .
$$

ayont les maxima, comme auparavant aupres de l'isotherme $T=T_{\text {iuw. }}$ (où $r=R_{1}$ ):

$$
\begin{aligned}
& \left.\max X_{\mathrm{rr}}\right|_{r=R_{1}}=\frac{20 G r^{\prime} \Delta R\left(R_{1}^{3}-R^{3}\right)}{3\left(5 R^{3}+4 R_{1}{ }^{3}\right)},
\end{aligned}
$$

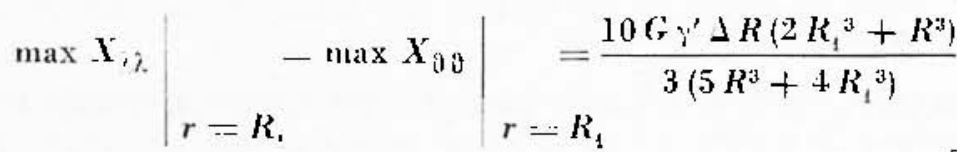

Entre max $X_{t r}$ ol max $X_{i, \gamma}$ existe dans ces cas la mèue liaison que celle clu cas de 2 :

$$
\text { max } X_{32}=1 / 3 \text { max } X_{\text {rr }}+109 g \% \perp K \text {. }
$$

Les fontions [ $13 \mid$ et $[14 \mid$ sont, en dépit de leur a]parence compliquée trissimples; dans l'intervalle avant le sens physicpue: $0<R_{1}<R$ elles n'ont ni maxina ni minima. Fn intuodusant la fonction

$$
R \quad-\frac{5+4 y^{3}}{5+1}
$$

d'un aroument $v=\frac{R_{1}}{R}$ de mesure, on a:

$$
=\underbrace{10 G \gamma^{\prime} R}_{3} M\left(\frac{R_{1}}{R}\right.
$$


et, d'après [15]:

$$
\max X_{\mathrm{rr}}=10 G \gamma^{\prime} R \cdot W\left(\frac{P_{1}}{R}\right)-10 / 3 G \gamma^{\prime} R\left(1-\frac{R_{1}}{R}\right) .
$$

La fonction $M(y)$ est representéc sur le lableau suivant:

\begin{tabular}{|c|c|c|c|c|c|c|c|c|c|c|c|}
\hline$y$ & 0.0 & 0.1 & 0.2 & 0,3 & 1). 1 & 10.5 & 0,6 & 0.7 & 0. H & 0,9 & $0 .(1)$ \\
\hline$H(y)$ & 0.200 & 0.180 & 0,162 & $0.1+5$ & 0.1217 & $0,11.4$ & 0,11978 & וור:0,0 & $0,0.57$. & 10.011 & 0.0000 \\
\hline
\end{tabular}

et illustre par la fig. 6h. On voit farilenent que, das les intervalles plus restreints, $M(y)$ peut tutre représentice d'une facon très simple: par exemple:

$$
Y(y) \geq 0,32\left(1-y^{\prime}\right) \text { pour } 0,9<y \leqslant 1,0\left(5700 \mathrm{~km} \leqslant R_{1} \quad 6350 \mathrm{~km}\right.
$$

avec une erreur $4 \%$, ou:

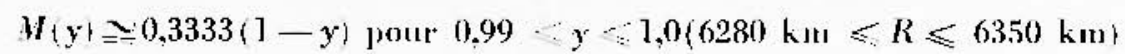

avec une erreur $s 1 \%$, on enfin:

$M(y) \supseteq 0.32\left(1-y-0,13(1-y)^{\circ}\right.$ pour $\left.\left.0 \leq y-0\right)(1)-R=6350 \mathrm{~km}\right)$

avec une erreur ne depassan pas esientiellenent la precedente.

Pour le probleme de la fissuration de lécorce on u'a besoin des très grandes profondeus; on pout délibérément se borner atu cas |20|. Alors, les fonctions déterminant la tension et la pression au point $r=R_{1}$, s'érirons:

$$
\begin{aligned}
& \max X_{\lambda_{2}} \cong 10 / 3 G r^{\prime} \cdot 0,32\left(R-R_{1}\right) \\
& \max X_{\mathrm{rr}} \cong-10 G_{\gamma^{\prime}} \cdot 0,01\left(R-R_{1}\right)
\end{aligned}
$$

cependant pour la deuxiome est preferable l'expression:

$$
\max X_{\mathrm{rr}}=-10 G \gamma^{\prime}(0), 01\left(R-R^{\prime}\right)+0,12 \frac{\left(R-R^{\prime}\right)^{2}}{R} /
$$


découlant de $\mid 201\} \mid /$. On se convaine facilement que la forme de la liaison entre $m a x X_{\lambda\rangle}$ et max $X_{r}$ reste inchangée (voir la formule [15] t. Mais une grande différence apparait entre la formule $[33 \mid$ du $\leqslant 2$ et la formule $[15 \mid$ de ce paragraphe. Là la valeur donné d'une des grandeurs $m a x X_{\lambda, i, m a x} X_{\mathrm{rr}}$ détermine complètentent la seconde. Ici, au conIraire l'une de ces grandeurs n'est pas déterminée par l'autre; on doit connaitre la profondeur de l'écorce formée à ce moment pour avoir la poszibilité de réaliser un tel calcul. C'est pourquoi le raisonnentent du 1ype du $\$ 2$ (voir la fig. ádl est ici impossil,le: le processus correspondant au refroidissentent "quasi-stationnaire" a un caractère complete-

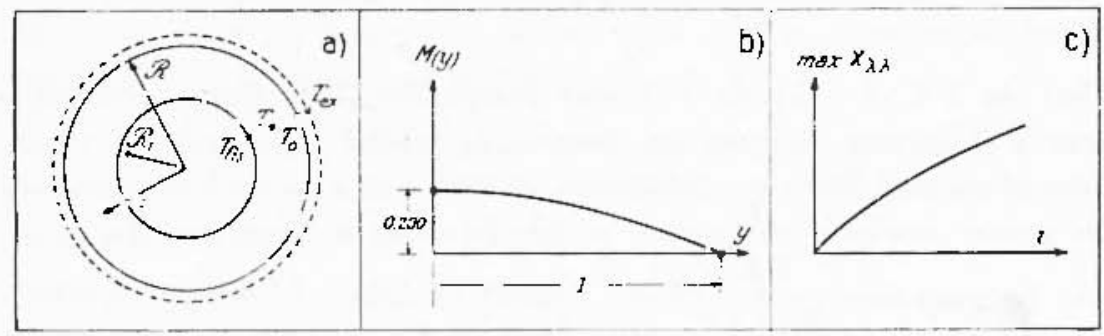

Fig. 6

ment différent de celıi gui correspond au refroidissement "isolhermique $n$.

L'endroit avec la plus forte tensions (- l'endroit oi commencera la fissurel est loujours pres de la surface $r=R_{1}$ a la profondeur de l'écorce mais le sens de variation de la plus forte tension en fonction de développenent de l'écorce est opposé all sens de cette variation dans le cas precedent: clans le cas $d u \leqslant 2$, cette tension a éte la plus considérable au monent de la formation de l'écorce, dans le cas considéré ici, celte tension est nulle au commencement de la formation et elle croit ares: l'épaisseur. l'our l'intervalle de temps pendant lequel l'écorce altonente d'épaisseur cette tension maximale est représentie. en fonction du temps par la fig. 5e clans le cas du processus " isolhermique " el par la fig. oc dians le cas du processus a quasi-stationnaire $n$ où $\gamma_{\text {totale }}=\gamma^{\prime} \unlhd R$. Enfin, on roil les cas intermédiaires sur la fig. Gol.

De tout rela, on peut tirer les conclusions sous forme dinègalilés, comme celles qui se trouvent a la fin du parayraphe précedent a qui ne sont applicable quaux cas sans influence de poids - comme ceux du $\$ 2$ (voir aussi $\$ 4$ ). Ces conclusions sont: 
1. Aussi longtemps que la grandeur $\perp R$ eroisiante, reste encore telle que la valeur correspondante de maxX satisfasse à l'inégalité: $\max x_{3 ;}=\frac{10 G \gamma^{\prime} \unlhd R\left[2(R-\Delta R)^{3}+R^{3}\right]}{3\left[4(R-\perp R)^{3}+5 R^{3}\right]}<\frac{F}{K-3 F}\left(K-10 / 3 G \gamma^{\prime} \perp R\right)$

aucune fissure ne se produit, parce que les contlitions [36] du 5 et [15] de ce paragraphe sont encore incompatibles. Au monent où

$$
\max x_{i i}=\frac{F K}{K-3 F}\left(1-\frac{10}{3} \cdot \frac{G \gamma^{\prime} \unlhd R}{K}\right)
$$

[36] du $\$ 2$ et [15] du $\$ 3$ sont compatilules, les fissures commencent à se former. Ce sont des fissures de second lype (voir $\$$ l), car, nes (formées) dans la profonteur, et non pas a la surface, elles ont les pentes marqués el presque perpéndiculaire à la surface du corps.

La rrandeur $\frac{F K}{K-3 F}$ etant d'apres [37] du $\$ 2$, toujours positive, et les valeurs de $\max X_{\gamma . t}$ et de $\frac{10 G \gamma^{\prime} \Delta K}{3 K}$ nulles au conmencement de la formation de l'écorce, l'inégalité $[23]$ est nécessairement valable lors de ce commencement. Enfin, la granteur $\perp R$ ne dépassant jamais $R$, cette conclusion s'écrit définitivement: lorsque:

$$
2 G \gamma^{\prime} R>\frac{3 F K}{K+2 F}
$$

les fissures du second type se forment.

2. Lorsque :

$$
2 G \gamma^{\prime} R<\frac{3 F K}{K-2 F}
$$

aucune fissure ne se forme el le corps considérí se solidlific sans traces de fissuration extròmes ou même internes.

\$ 4. Résumé dess résultats du calcul. Quelques applicutions. Théorie de l'écorce terrestre. -. Les résullats du $\$ 2$ et du $\$ 3$ ne doivent pas crèer l'impression que tout dépend du caractère du processus. Au contraire, c'est le fail de la prépondérance du terme $\gamma$ ou du lerme $\gamma^{\prime} \perp R$ dans l'expression $[8 \mid$ du $\$ 3$ qui détermine l'apparition des fis- 
sures du premier ou du second type. Mais, d'autre part, c'esi seulement pour un processus non "isolhermique" que l'on peut poser la question de celle prépondérance; pour un processus "isothermique " le terme $\gamma^{\prime} \Delta R$ érale toujours zèro. Alors, en résumant:

I. Durant un refroidissement "isothermique" ou plus genérale. ment a quasi-stationnairs", mais en ce deuxiems cas sous condition d'une valeur de $\gamma$ considerable devant $\gamma^{\prime} \Delta R$, ce sont exchtsivement les fissures du premier type qui peuvent se former.

II. Durant un refroidissement "quasi-stationnare" et non "isothermique", sous condition d'une valeur de $\gamma^{\prime} \Delta R$ considerable devant $\gamma$, ce sont les fissures du second type que se forment pxclusivement ou aucune fissure ne se? Jorme. Enfin:

III. Durant un refroidissement "quasi-stationnaire" où aucum? des valeurs: $\gamma$ 't $\gamma^{\prime} \Delta R$ ne prevant sur lautre. les fissures de tous les types peuvent se former.

Il est à souligner encore une fois que les fissures se forment seulement ì contition que les consiantes physiques du corps en question satisfassent aux inégalités cruciales - [39] et $[40]$ du 2 clans le cas I el [26] du $\$ 3$ dans les cas 11 et III. En outre, on ne doil pras oublier que les fissures du second yype naissent aussi de causes purement mécaniques, et non exclusivement de causes thermiques. C'est le cas, par exemple, pour les bombes volcaniques, où plusieures des fissures du second type sont formés au moment où la homlic se casse en tombant sur le sol.

I a simple théorie cléveloppée dans cette étude peut avoir des applications nombreuses. Mentionnons d'ahord les fissures sur la surface des bombes volcaniques (cerlain types "à croutc de pain ", elc.). Ies deux types de ces fissures découverts par l'auteur (') peuvent ètre maintenant examines qualitalivement, mais non seulement du point de vue purement statistique. Mais si l'hypothèse du processus a quasistationnairen suffit totalement pour la description mathèmatique du refroidissement de l'écorce terrestre, ce n'est pas toujours le fait pour les bombes volcaniques - voir aussi [\%. I a lléoric tléveloppée peut êlre également appliquée à la fissuration des corps artificiels en voie de refroidissement. Le résultat le plus général est le méme que le procide toujours applique dans la pratique: un refroidissement le plus lent possible taussi voisin qu'il se peut d'un processus "isolhermirtte $n$ i rst le meilleur procéclé pour éviler la fissuration. Cöncidant 
sur ce point avec la pratique, la lhéorie donne plus: les formules du calcul des tensions, les incigalités cruciales (que nous venons de menlionner) chteminant le commencement de la fissuration dins les cas divers, etc. En oulre, la question qui n'est pas apparement de yrand intérêt pratique, - la question de la fissuration des scories contées des hauts-foumaux, peut être éralement illustrée par celle théorie. On peut multiplicr de pareils exemples presque à l'infini.

Or, nous laissons toutes ces possibilite's d'application pour un examen ulterieur et d'autres endroits. C'est la question du refroirlissement de l'écoree terrestre à laquelle nous domons ici la place.

Cependant, comme nous l'avons fait remarquer dans les paragraphes précerlents, la théoric développée n'est pas dircctement applicable qu'aux cas où l'influence de poids est néglizeable. Pour les corps ayant, comme la Terre, les rayons de milliers de kilomètres, ce n'est pas le cas et les poids cloivent être introchuts. Si l'on considère la solidification d'un corps jusqu'au centre, la seule possilrilité ent d'introduire les poids dans l'éfuation $|2|$ et de resoudre rette équation avec ce terme complémentaire. Cette méthode, étant irreprochahle: du point de sue théorique, n'est pas ni la plus simple, ni la seule possible. Si nous nous bormerons aux cas de l'feorce pen épaisse en comparaison avec le rayon - et c'est le cas de la Terre - on peut introduire l'influence de poids simplement sous la forme d'une cor.

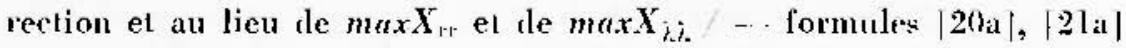
du $\leqslant 2,|13|,|14|$ du $\leqslant 3$ ou de $X_{\text {rr }}$ et de $X_{\lambda, \ldots}$-..- formules $\left.\mid 20\right]$. $[21 \mid$ du $\leqslant 2,|11|, \mid 12]$ du $\$ 3$ considérer correspondement:

$$
\max X_{\mathrm{rr}}-\rho \Delta R \text { et max } x_{i}-\rho / 3 \Delta R
$$

it

$$
X_{\mathrm{rr}}-\varrho(R-r) \text { et } X_{\lambda \lambda}-\vartheta / 3(R-r)
$$

Cette correction ne donne naturellement pas une exactitude parfaite. Mais, si les formules des parawraphes précedents, appliquées a l'écorce lerrestre anèment ì des erreurs considérables, les valeurs calculfes à l'aide de la correlation indiquée, ne différent pas des valeurs exactes de plus de $1 \%$, même pour une écorce de $100 \mathrm{~km}$ d'épaisser.

Le premier fait important découlant de la théoric exposée est le fait que l'écorce en voic de: refroidissenent a fite tendure, et non pas comprimíe conme l'a affuné la lbéorie dite de la contration. Sauf les cas des phases solides de l'eau et des fontes speciales, - cas où 
$\gamma<0$-, "as exceptionnels et éloinnés de l'écorce réelle, pour tout corps réel, avec $\gamma>0$ l'existence de la tonsion est, d'après le caleul exposé dans $\$ 2$ et $\$ 3$, hors de doute. C'est poure[uoi notre caleul exclut complitement la theorie de la contraction.

Olbjecter que l'écorce n'a pas été forme complètement et atutour de toute la Terre, revient à dire, quion emploie le mot "écorce" dans un atutre sens que celui applicpue plus hat. On peut affimer naturellement qu’à certain moment une pré-écorce a existé ne s'étendant peutêtre pas sur toute la surface du vlobe. Mais au moment de l'apparition de la phase liquide de $H, O$ sur cette surface, - et ce moment a cu lieu bien avant les périodes géologriques imême avant le précambrient-- l'écorce existe, convrant complètement et sans lacunes toule: la surface du plolie.

Et, comme le montre le calcul exposé, dès le moment de la formation de lecorce, l'etat de tension tangentielle a surgi dans la surlace. Les pressions, heacoup plus petiter que les tensions, ne peuvent se former rue dans les clirestions maliales, s'opposant aux poids. L'écoree entournant le glohe, ne pousant être tendue que tang̣entiellement, est. de fait, tendue et l'existence virtuelle des pressions vertirales fradiales| ne cliange ricn à ce phénomène.

Alors, peu importe, si la prétcorce wegnerienne a existé ou n'a pras cxisté, le moment venu, l'écorce at propre sens du mot sc forme aulour de tout le irlobe terrestre et se forme soumise à l'état de tensions.

Sculs les métaux possèdent des résistances preu differentes à la pression el à la tension. Pour les roches ce n'esl pas le cas: ces dernièrs supportent très facilement des pressions enormes et rompent sous des tensions relativencm faibles. Pour cette raison il est excessivement improbable que l'écorce ail pu résister atux tensions en question, spécialement - ce qui a été le cas - aux températures proches de celle do la lusion.

Tout rela nous permet de soulenir une thiorie de l'écorce ayant pour but d'expliquer la formation des rontinents el des montapues, - Ihéorie basée sur l'existence des tensions. Cette lléorie proposée par nous dis aran la wuerte, est formulee en ses difterentes parties dans plusientes publications dont trois sont déja citées: qucle[ues unes des autres seront mentionnes plus has. Voici les traits les plus imporlants de celte théorie de l'orogénise.

On admel au départ que la Terre pendant une éprouge de son bistoire tiloignée de nos jours elail liquirle el fu'clle est en se refroi- 
dissant parvenue à son état actuel. Le refroidissement spontané, dû aux réserves d'énergie non infmies dans le noyau, a donné lieu à la formation de l'écorce. Cette dernière, étant tenduc, n'a pu que se fractionner. Les morceaux ainsi formés, correspondant en état non tendu à un rayon moindres que celui du noyau et n'ayant pas possibilité de conserver leurs formes, sont devents plissés parce que des parties de sphères de rayons différents ne son pas "applicalules" (en langage mathématique) les unes sur les autres [voir ( ${ }^{51}$ ), où se trouvent les figures explicatives]. On peut considérer le refroidissement du Glolie soit comme un processus mathénatifricment monotone, soit avec un rechauffement au moment de la formation de l'écorce (voir 15)); cela ne change rien au fait mécanique que les variations des volumes de l'écorce et lu noyau n'étaient pas el ne sont pas iquivalentes en grandeur. Comme le calcul le montre, la variation du volume de l'écorce est plus considérable que celle du volıme du noỳau. Même pour le cas d'une extrême lenteur du refroidissement - processus "isolhermique n) ce rétrécissement thermique de l'écorce relativement au noyau est facilement établi — voir $\$ 2$. Dans les cas des processus plus "lirusques" ce fait se met encore plus on relief.

On ne peut pas cependant penser que ce sont les raisonnements mathémaliques et mécaniques qui jouent le rôle principale lorsqu'on décide d'affirmer ou de nier une théorie liée à la réaliti. Ce sont plutôt les conclusions qu'on peut tirer de la théorie et leur councidente suffisante on insuffisante avec les olservations qui léterminent la decision. Et nous avons essayé de clonner plusieures preuves: mëme notre theorie nous a permis de découvrir les faits nouveatix. Sans entrer dans le délail, énmmérons quelıtes uns de ces réstltats tiris de notre theorie et la confirmant:

Li Relalion entre l'allitude moyenne et la stuperficie des conlinents $(52,53)$.

2) Distribution des voleans sur la surface de la Terre $\left({ }^{4}, 55\right)$.

3) La théorie de volvanisme ("iti).

4) Ressemblance des cotes atlantiques africaines et sul-américaines ( ${ }^{57}$ ).

5) Stades du développement du plissement (57, 58).

6) Lois générales du relief terrestre ("50).

7) Les limites les blocs continentaux (60).

8) La quantité de matière éruptéc par les voleans en liaison avec la distribution de ceux-ci (it). 
9) La position du pli le plus important sur un continent de forme approximativement triangulaire $\left({ }^{82}\right.$ ).

10) Le critérium d'orogénèse $\left({ }^{63}\right)$.

Le nombre des publications faites par les géologues et les géophysiciens sur le sujel de l'écorce terrestre est énorme. Quelques indications peuvent se trouver dans nos travaux susmentionnés. De plus, nous avons publié une hilliograplie spéciale sur ce sujet (voir "it)i. Nous ne Ia réproduirons pas ici. Seules les pululications sur le regrime thermique de l'écorce sont en parties citées - voir de $\left(^{3}\right)$ jusqu'à ${ }^{i 2}$ ).

Revenant pour la dernière foi aux résultats de $\$ 2$ et $\$ 3$, soulignons que la valeur de tout ce calcul est - semble-t-il - plus qualitative que quantitative. Etant donné que toutes les constantes phy. siques nécessaires pour le calcul numérique, sont connues avec peu de précision, il semble qu'un tel calcul soit prématuré en dépit de l'exacti. tude du calcul theorique, - exactitude plus que suffisante pour les besoins des sciences de la Terre. Cependant, sans insister sur l'importance des chiffres sousmentionnés, nous citons, à titre d'exemple, le calcul stivant très approximatif et très court.

Supposons pour Ia Terre un processus de refroidissement "quasistationnaire" et l'écorce peu épaisse au moment de l'apparition des fissures, ce qui concorde évilenunent avec la réalité. Cela clit, on peut appliquer les formules $\lceil 7 \mathrm{za}]$ et $[21\}$ du $\$ 3$. En portant la valeur de $\gamma^{\prime}$ d'après [7a] dans $[2]\}$, on trouve (en cas de l'alsence de poids):

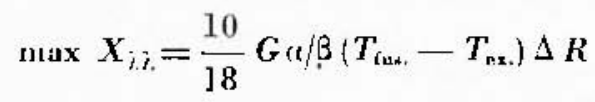

Pour la nêne raison is $R$ nötant pas considérabliè en cfs de la presence de poids on peut appliquer la correction $[\mathrm{A}]$ et poser simplement :

$$
\max \sec \mathrm{X}_{j, .}=-\frac{18}{18} G_{a / \beta}\left(T_{\text {fus. }}-T_{\text {ex. }}\right) \Delta R-\mathrm{d} / 3 \Delta R
$$

au moment de là naissance d'une fissure. Les conditions [D] et [36] du 2 étant réunies, on a:

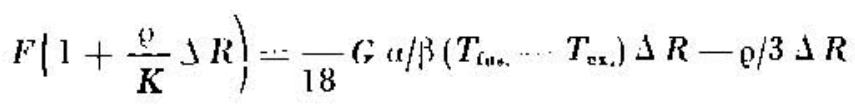


Essityons de calculer à l'aide de cette formule [E] l'épaisseur de l'écorce 1$\rfloor R$, au moment de la naissance des fissures. Faute de connatitre des valeurs exactes des constantes physiques pour Ies cas considerés, le calcul sera plus qu'approximatif. Les trandeurs $G$ et $F$ varient entre les valeurs:

$$
\begin{aligned}
& G \cong 100000 \mathrm{~kg} / \mathrm{cm}^{2} ; \\
& F=200 \mathrm{kgr} / \mathrm{cm}^{2}-100 \mathrm{k \not ̣} / \mathrm{cm}^{-} \text {ou même encore moin }
\end{aligned}
$$

et :

$$
\alpha \supseteq(1 / 5000)^{\circ} ; T_{\text {fun. }}-T_{\text {ex. }}=1000^{\circ}-1500^{\circ}
$$

Le plus difficile est le calcul de ;'. Dans notre publication déja citée $1^{2}+$ nous avons donné le détail sur le caleul numérique de $\beta ;$ on peut également $y$ trouver le tableau des valeurs dérivées températures, temps, ete.] pour diverses valeurs de [3. Il semble cependant que la valeur $\beta=300-100 \mathrm{~km}$ est la plus convenable. En l'arloptant, on trouve: $\Lambda R=30 \mathrm{~km}$ (pour $\beta=200 \mathrm{~km}$ ) ef $\lrcorner R=2 \mathrm{~km}$ (pour $\beta=150 \mathrm{~km}$ - valeurs qui sont en principe admissible. Pour le détail voir l'article cité,

Pour terminer, répétons encore une fois que le résultat principal de cette etude est lc résultat qualitatif sur l'existence de l'état tendu de l'écorce ( $\$ 2$ et $\$ 3$ ); l'incertitude sur les valeurs des constantes, ne permet pas un calcul numérique exact.

\section{RIASSUNTO}

L'Autore calcola le tensioni nol mantello terrestre, durante la formatione delle spaccature "primarie" nella crosta terrestre. Sulla base del teorema di Stokes, rghi ha potuto prescindere dalla necessiti di prendere in considerazione Fipotetica distribuzione dolle temperature nellinterno della Terra.

\section{SUMMARY}

In the present paper the author calculates the tensions in the earthis mantle during the formation of the primary fissures; on the base of the Stokes's theorem he has eliminated the necrssity of taking into consideration the hypothetical distribution of the temperatures in the earth's interior. 


\section{LES ALITEURS CITES}

(1) Hatschisski M., Observution sur la morphologie des bombes volcriniques. C.R.S. de la Sor. Génl. de Fr., II. 10, 1. 149 (1951).

12) Hatschisiki M., Surface des bombes volcmiques on tant que modelc approximatif de la surface teirestre. Rev. Scientifique (1952).

(i) KeLvix (Lord), On the secula cooling of the earth. Roy. Soc. Edinburgh Trans., 23, p. 157-69 (1862).

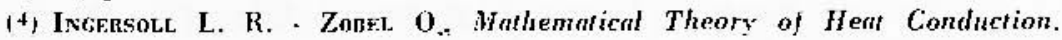
P1. 88-96 (1913).

(5) S'ICER H. C., Tables of temperature, genthermal gradient and of "n nonradiuntite earth. Geol. Soc, Amer. Bull., 18, p. 75.91 (1937).

(15) Jovenus; T. S., Heat conduction in dissimilar rocks and the use of thermal models. Geul. Soc. Amer. Bull., 47, p. 87-100 11936).

(7) Houves A., Ralinativity and the eath's thermal history. Geol. Matg., new ser, Decalde VI, 2, p. 102-12 (1915).

(1) Ausus L. H, Temperatures at moderate depths within the earth. Washington Acad. Journ., 14, 1. $459-72$ (192-1).

(!) Jefresis H., The Earth., p. 148, 2 d ed. Cambridge (1929).

(ii) JyFfkES H., Philosophisal Magazine, 2, p. 833 (1926).

(11) JfFruess H., The Deformution of the Earth due to Unsymmetrical Cooling. Geoph. Supp.to the M.N. of the R.A.S., vo. III, p. 53 (1936).

(12) Bullat" E. C., H.N.R.A.S, Geophys. Suppl, 4, p. 534 (1939).

(1:3) WolfF F. vox, Das Temperaturgesetz in der Eralkruste. Jatırb. Hallescher Verband Erforschung der mittcldeutsrlon Bodensthätze, 7 ineue Folget, 1. I \{1928\},

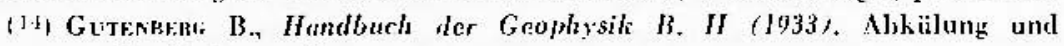
Tentperatur der Erde. p. 33.

(15) Gutenbrac B., Physics of the earth. New York and London (1939) VII Internal Constilution, Ch. VII. The cooling of the earth atud the temperature in its interior.

(Iti) Curasan S. - Pluge. A. T. The eletric and magnetic state of the interior of the carth... Roy. Sor. London Pluil. Trans. (A), 229, 1. 427-60 (1930).

(ii) Rukjtake T, A Note on the Temperature - Distribution teithin the Earth. Bull. of the Earthqumake Researeh Inst, XXVII, part I-h, p. 17 (1949).

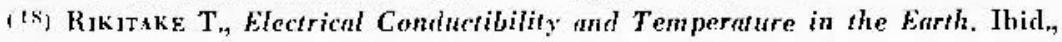
XXX, part I, J. 13 11952).

(1i) Buach F., Flove of heat in the Frant Range, Colorato. Bull. Geol. Soc. of Amerisa, 6I, p. $567-630$ (I950).

(-11) Verhoncex I., Volcanic heat, Amer. Journ of Sti, 244, p. 745-71 (19:46).

(21) Venuonger I, The Adinbatic Grodient in the Mantle. Trans. Anter. Geophys. Inivn, 32, p. 41 11951).

i--) VaLte P. E., Sultamento di temperatura nel Mantello delli Terra. etc. Annali di Geofisien, val. It, H. 175 (1951).

1 an Valte P. E., Sul gradiente adiabatico di temperatura nellinterno della terrn. Iljid, vol. F, n. l, p. 41 (1952).

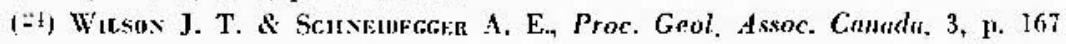
11950). 
(ii) Schnithager A. E., Physical aspects of the contraction hypothesis of arogenesis, Canad. Journ, of Plisyca, 311, p. 11 t.lan 1952).

(26, Davisox C., Pliil, Trans. A, 178, p. 231 (1887).

(*i) Dans G. G. Phil. Trans, A, 178, p. 2.12 (1887).

(28) Jeafneys H., Earthquakes and Momeans. Methuen, London (1950).

(2) Corlous J., Tensions engendries dans le globe terrestre par son refrodissement, Ant, Géoph., T. I., fass, 2 tMars 19:1t.

(30) Raylenih Lord., Plit. Mag., 32, p. 529 11916).

(31) Lowan A. $\mathrm{X}$, On the Conling of a Radionctive Sphere. Pluys. Rev. vol. It, sec. ser., p. 769 (1933).

(a2) Lowax A. N., Note on the Cooling of a Radionctive... Sphere. Amer. Juuru. of Hath., vol. INI, p. 2.4 \{1934).

(33) Lowax A. N, On the Cooling of the Earth. Ibid, vol, LVII, p. 17 (193.).

(3+) Slichteн I.. B. Cooling of the earth. Bull. Geol. Sor. Aner., vol. 52. p. 56$]$ (19.11).

(35) EnRy W. 1). Siznifirance of Radionctivity in Geophrsics, thermal histors of the Earth. Trans. Amer. Geoph. [Tn., vol. 30. p. 171119.49$).$

(30) Gextry F. M., Science, D. 1811, 70, p. 332 11929).

(37) Kcits W. und Rutmans A., Gber den Zustand des Erdinnera und srine Fintstehung ans einen homogenen bratstand 119.41$)$.

(38) Limagnve J. H. F, Contraction of the earth. Proc. of the Kkl. Nederl. Ak. v. Wetenschitp, ser. B, n. 2, p. 105 (1952).

(at9) Wasturysski J., Studies in Hydrodyuamirs and Struture of Stars and Planets. Astroplyysica Norvegica, vol. 1, Osto 119467.

(40) Pekeris C. I., Thernal convection in the interior of the earth. Mouty Not. Roy, Astronom. Sor., Geoplyys. Suppl, 3, p. 3.13 (1935t.

(11, Veninf Mensesz F. A., Major tectonic phenomena and the hypothesis of convertion currents in the Earth. Qularterly Journ. of the Geol. Soc, of London. vol. 103 , p. 191 (19.181.

(42) Vexus: Mejess? F. A., Convention currents in the Mantle. Trans. Amer. Geophys. Inion, vol. 32, n. 1, p. 531 (1951).

(43) Stokes, Proc. Camh. Phil, Soc., 8, p. 672 (18.49).

14-) Tusst:rand, Traité de méconiqne celeste. $\$ 119$, t. II, Paris $1189 \mid$ t.

14j) Matschinski M., La formation des rontinents et des forres géodynami. ques. C. R. Ac. Sci, de France, t, 230, p. 1882119501$).$

(4ค) BÉxsto II., Les tourbillons cellulaires duns un nappe liquide. Rev. gén. des Sei., XI, n. 23, p. 1261-71 115.XI1.1900), p. 1309-28 (30.XIl.19001. - Les tourbillons: cellulaires dans une nappe liquide transportante do la chalpur par convection en régime permanente. Ann, de Chen, et de Plıys., ;me seric, t. XXIII, p. 62-114 119011.

1.17) Gejser und Scheel. Handburh der Physil; Band VI, p. 463 , Berlin Springer (1928).

(48) SAINT-VENANT, C. R. Ac. Sci, de France, t. 70 , p. 368 et 173 1870): ihid. t. 73, p. $86,1098,1181$ (1871).

(49) Colloquinm on phastic flow and deformation within the earth, n. 1, vol, 32 of the Trins. Amer. Geophys, Union 6 (1951).

(50) Misfs von R., Göttinger Narlur. 11913). 
(31) Matschixsk, M., Sur Porigine des forces orogénétiqnes. Calhiers Gëol. de Thoiry, n. 4, p. 59 (1951).

(52) Matschinski M., Relation entre Tultitude moyenne et la superficie des continents. C. R. Ac. Sci. de France, t. 233, p. 492 (1951).

(:i3) Mitschinskı M., Allitude moyenue des continents et forces géodynumiques. Rev. Géomorph. Dyu., n. I, p. 157 (1951).

(5) Matschinsk I M., Sur la distribution des volcans à la surjace de la Terre. C. R. Ac. Sri. de France. t. 233 , p. 669 (1951).

(59) Matschinsk I M., Données numériques sur la distribution des volcans des bords de Rocean Pacifique. Rev. Gáomorph. Dyn., n. 6, p. 276 (1951).

(joj Matscinnski M., Quelques remarques sur les couses d'érnjtion et sur le röle du gaz degagé por rechunffement. Calıiers Gèol. de Tlıoiry, I. 9, p. 77 (1951).

(nit) Matschinski M., Ressemblance des câtes atlantiques africane et sudaméricuine et dévelopjement du plissement dun continent isole. C. R. Ac. Sri. de France, t. 233, p. 1653 (195]).

(5s) Matscunski M., Studes de l'éculution du plissement sur un continent isole. Gahiers Giénl. de Thoiry, n. 10, p. 171 (1952).

(iii) Matschixsk M., hois générules dn rélief terrestre. C. R. Ac. Sti. de France, t. 234 , p. 1192 (1952).

(bio) Matschissk $\mathrm{M}_{\text {. }}$ Les limiles des blocs rontinentan. C. R. S. de la soc. Géol. de France, n. i, p. 105 11952).

(131) Matsciunski M., Distribution des volcms sur ln surface de la Terre. C. R. S. de la Sor. Géol. de France, 11. 12, p. 218 \{1952j. Errata: ibid, n. 1t, p. 314 (1952).

("iz) Matschinsk] M. Missement itu continent de forme approximativenent iriangulitire. C. R. Ac. Sci. de France, t. 23.1, p. 2473 (1952).

(6) Matscinssk 11 , Le critérium enlérien de la stubilité de blors continen. taux, critérium torogènèse. P’roc. Kkl. Nederl. Ak. Wetensch. ser. B. 55, n. 4. p. 111 (1952).

(fil) Matschinsk M., Bibliographie sur les questions de l'érore terrestre et de Misostasie. Rev. Géomorph. Dyn., 11. 6. Supplement. p. 1. (1953). 